\title{
Coulisses
}

Revue de théâtre

42 | Printemps 2011

Racine : Théâtre et émotion

\section{La Thébaïde en examens (1768-1824)}

Jean-Noël Pascal

\section{OpenEdition}

Journals

Édition électronique

URL : https://journals.openedition.org/coulisses/542

DOI : $10.4000 /$ coulisses. 542

ISSN : 2546-9460

\section{Éditeur}

Presses universitaires de Franche-Comté

\section{Édition imprimée}

Date de publication : 15 février 2011

Pagination : 17-29

ISBN : 978-2-84867-316-5

ISSN : 1150-594X

Référence électronique

Jean-Noël Pascal, «La Thébaïde en examens (1768-1824) », Coulisses [En ligne], 42 | Printemps 2011 , mis en ligne le 30 novembre 2016, consulté le 29 décembre 2022. URL : http://

journals.openedition.org/coulisses/542 ; DOI : https://doi.org/10.4000/coulisses.542 


\title{
La Thébaïde en examens (1768-1824)
}

\author{
JEAN-NOËL PASCAL \\ Professeur à l'Université de Toulouse - Le Mirail
}

Les premières éditions savantes des auteurs français appelés bientôt à former le corpus classique dans l'enseignement réorganisé par l'Université impériale - dont peu ou prou, à travers de multiples réformes, l'essentiel s'est perpétué jusqu'à nos jours - apparaissent peu après 1760. La démarche, sous ses deux aspects d'établissement du texte et de commentaire, n'est pas sans analogie avec l'entreprise encyclopédique contemporaine. Elle entend, du moins, faire le point sur l'état des connaissances et comporte un indubitable aspect taxinomique : il faut inventorier et classer les chefs-d'œuvre du siècle de Louis $\mathrm{XIV}$ en les accompagnant de commentaires destinés à marquer leur place et à analyser leurs beautés. Autrement dit: expliquer méthodiquement et valider rationnellement le consensus qui s'est établi à leur propos.

Entrer dans le détail de l'histoire de cette démarche de hiérarchisation de la production littéraire nous entraînerait beaucoup trop loin ${ }^{1}$. D'abord un peu désordonnée et essentiellement justifiée par des motivations esthétiques jusqu’à la Révolution, elle devient méthodique et politique sous le Consulat et l'Empire, puis systématique et pour ainsi dire industrielle - les évolutions techniques de l'imprimerie et l'élargissement du lectorat n'y sont pas pour rien - et systématique à partir de la Restauration. C'est évidemment dans le secteur de la poésie dramatique qu'elle est la plus facile à discerner, même si elle touche aussi progressivement d'autres domaines: le répertoire qui s'est constitué, depuis 1680, se subdivise en ouvrages « du premier ordre » - généralement ceux de

1. Pour des précisions voir J.-N. Pascal, «Sur les préfaces des éditions commentées des CEuvres de Racine, de Luneau de Boisjermain à Étienne Aignan (1768-1824) », dans L'Art de la préface au siècle des Lumières, collectif dir. par Iona Galleron-Marasescu (Rennes, PUR, 2006) et «Le statut de l'histoire littéraire dans l'enseignement secondaire à la fin du XVIII et au début du XIXe siècles", dans L'Histoire littéraire et son enseignement, $\mathrm{n}^{\circ}$ spécial de L'École des Lettres (1994). 
Corneille, Molière et Racine -, « du second ordre » - ceux de leurs successeurs de la charnière entre le XVII ${ }^{e}$ siècle et le XVIII e et ceux du temps des Lumières - et même, à partir de 1820, « du troisième ». Dans le paysage éditorial, se multiplient, à partir des années 1760 puis surtout - en trois vagues assez rapprochées - dans les années 1780, 1800 et 1820 - des séries aux intitulés variés (Bibliothèque du théatre français, Chefs-d'cuvre du théâtre français, Répertoire du théatre français...) à peu près toutes organisées selon le même schéma: les Euvres complètes des auteurs indiscutables sont accompagnées des Euvres choisies ou même des pièces isolées d'autres dramaturges déjà disparus ou encore vivants, classées par genre (tragédies, comédies, bientôt drames, opéras, mélodrames...) et selon un ordre à peu près chronologique. Et même si certaines de ces séries se contentent parfois, notamment les moins prestigieuses, de reproduire les ouvrages, plusieurs les accompagnent d'un appareil impressionnant de notes, d'observations, de commentaires, de préfaces et d'examens, selon un modèle apparu dès les années 1760 .

Placées souvent ainsi en position - si l'on permet - de locomotives pour des entreprises de librairie de grande ampleur, les CEuvres dramatiques complètes des auteurs de la triade classique - et bientôt de Voltaire tout aussi bien ${ }^{2}$ connaissent aussi une spectaculaire carrière autonome, sous la forme de publications commentées par des critiques souvent fameux. Dans le seul cas de Racine, qui nous intéresse ici, on relève au moins quatre éditions de ce type :

- la série des Euvres de Jean Racine avec des commentaires, par M. Luneau de Boisjermain (Paris, Imprimerie de Louis Cellot, 1768), forte de cinq volumes in$8^{\circ}$, dans une présentation typographique luxueuse, avec de très belles illustrations de Gravelot, disponible en reliure d'éditeur et complétée par deux volumes d'CEuvres diverses de Jean Racine enrichies de notes et de préfaces (Londres, 1768) ; cet ensemble 3 fut reproduit, avec la mention de «seconde édition», dans une présentation beaucoup moins somptueuse, à l'époque thermidorienne (Paris, de l'Imprimerie de Pougin, 1796-an 4e), le seul Commentaire de Luneau de Boisjermain (et de ses collaborateurs) ayant donné lieu à une publication au petit format sans les textes de Racine dès l'époque de la première parution (Commentaires sur les xuvres de Jean Racine, par M. Luneau de Boisjermain, Paris, Panckoucke, 1768);

2. On rappellera que c'est le Théâtre de Pierre Corneille avec des commentaires (1764), de Voltaire justement, qui lance la mode de ces éditions commentées. C'est probablement cet ensemble imposant qui provoque l'apparition des CEuvres de Jean Racine avec des commentaires (1768), de Luneau de Boisjermain, et des Euvres de Molière avec des remarques grammaticales, des avertissements et des observations sur chaque pièce (1773) de Bret.

3. Ce n'est pas ici le lieu d'expliquer longuement que tant le choix du titre que le luxe de la présentation et l'existence d'une version séparée du Commentaire de Luneau de Boisjermain s'expliquent par le désir de cet éditeur de calquer le modèle du Théatre de Pierre Corneille avec des commentaires, en faisant subir à Racine le même traitement que Voltaire avait imposé à Corneille, dénigrement - ici ou là - compris... 
- la série des Euvres complètes de Jean Racine avec le commentaire de $M$. de La Harpe (Paris, Agasse, 1807), forte elle aussi de sept volumes in- $8^{\circ}$, avec de splendides illustrations de Moreau le jeune; cet ensemble, mis au point par Germain Garnier après la disparition de La Harpe, mort en 1803, semble avoir été entrepris en 1795-1796 d'après l'Avis du libraire, mais pourrait bien être légèrement plus tardif et faire suite à la réimpression de l'édition de Luneau de Boisjermain contre laquelle le fameux critique polémique violemment et avoir été rédigé dans les marges de la publication du Lycée ou Cours de Littérature, commencée justement en 1799 chez Agasse ; il connut apparemment une publication rapide au petit format (en huit volumes in-12, par les soins de Mac Carthy) et le théâtre seul fut réédité 4 avec la mention d'«édition revue et corrigée » et les figures de Moreau (Théâtre complet de Jean Racine avec le commentaire de M. de La Harpe, Paris, Verdière, 1817);

- la série des CEuvres de Jean Racine avec des commentaires, par J. L. Geoffroy (Paris, Genets jeune, 1808), forte de sept volumes in $-8^{\circ}$, agrémentée de belles illustrations de Stéphane-Barthélemy Garnier, complétée au même format par les CEuvres de Louis Racine (Paris, Le Normant, 1808) en six volumes ; cet ensemble très érudit - notamment en ce qui concerne l'indication des sources antiques - polémique volontiers contre les jugements de La Harpe, en prenant à l'occasion la défense de Luneau de Boisjermain ; il semble n'avoir jamais été réimprimé ;

- la série des CEuvres complètes de J. Racine avec les notes de tous les commentateurs, auxquelles ont été jointes de nouvelles notes, et des études sur Racine, par M. Aignan, de l'Académie française (Paris, Dupont, 1824), forte de six volumes in- $8^{\circ}$ et ornée d'illustrations de Moreau le jeune ; cet ensemble joue sur deux tableaux : il vise à être une édition variorum, à la manière de celle d'Aimé-Martin, parue en 1820 , tout en laissant à l'éditeur - dans les «études » qui tiennent lieu des habituels examens - l'espace de jugements personnels ; il semble n'avoir jamais été réimprimé.

Ce ne sont évidemment pas les seules éditions de Racine parues en ces temps-là : déjà très fréquents avant la Révolution, les tirages - prestigieux ou non, dans tous les formats - du Théâtre de l'auteur de Phèdre se mettent à pulluler - certaines années, il en sort plusieurs - dès après Thermidor. Certains ne manquent pas, outre l'intérêt purement bibliophilique, de mériter la curiosité de l'érudit. Petitot, par exemple, l'éditeur du premier Répertoire du Théâtre-français, donne en 1820 une édition ${ }^{5}$ munie de quelques notes consacrées aux «imita-

4. Il ne s'agit pas vraiment d'une réédition : on semble avoir assez artisanalement utilisé le reste de l'édition princeps en y opérant quelques ajouts ou substitutions de cahiers au début.

5. Euvres de Jean Racine avec les variantes et les imitations des auteurs grecs et latins, publiées par M. Petitot éditeur du Répertoire du Théâtre-Français, Paris, Stéréotype d'Herhan, 1807, 7 vol. in- $8^{\circ}$. On notera que si le Répertoire était destiné à compléter les éditions in- $8^{\circ}$ des grands dramaturges français, Petitot n'a donné son propre Racine qu'après sa série consacrée aux auteurs du 
tions des auteurs grecs et latins", et Aimé-Martin offre en 1820 au public savant une série ${ }^{6}$ qui se veut objective, dans laquelle il ne s'accorde qu'une place très restreinte (quelques notes érudites ou informatives), se contentant de compiler les annotations et commentaires antérieurs.

Ce ne sont pas, non plus, les seuls Commentaires - pour l'essentiel déjà anciens - disponibles : les Remarques de grammaire sur Racine de l'abbé d'Olivet sont parues en 1738, suivies par leur réfutation due à l'abbé Desfontaines (Racine vengé, 1739) ; les Dissertations sur le progrès du génie de Racine de l'abbé Nadal remontent aussi à 1738 ; les Remarques sur les tragédies de Jean Racine, longues et minutieuses ${ }^{7}$, de Louis Racine, dernier fils du grand dramaturge, ont été publiées en 1752. Seules les Études de la langue francaise sur Racine ou Commentaire général et comparatif sur la diction et le style de ce grand classique (Paris, Belin-Le Prieur, 1818, 2 vol.), du grammairien Fontanier, appartiennent à la période que nous avons choisi de considérer.

Bref, l'œuvre de Racine est amplement évaluée, critiquée, écrasée sous la gangue d'un commentaire admiratif qui répète sous tous les tons que l'auteur de Phèdre est le premier des poètes français. Seule la tragédie de ses débuts échappe, partiellement, à cet embaumement dans la louange et c'est la raison qui nous a poussé à vouloir regarder d'un peu plus près quel jugement global les responsables des premières éditions savantes en portaient dans leurs examens et autres péritextes analogues.

\section{Luneau de Boisjermain ${ }^{8}$ (1768)}

Dans le tome I de la belle édition des Euvres de Jean Racine avec des commentaires, La Thébaïde est précédée d'une «Préface des éditeurs » qui rappelle au départ combien il est « important, pour les progrès du goût, de voir de quel point est parti l'auteur de Phèdre et d'Athalie» (p. 3) : c'est suggérer clairement que la carrière du dramaturge doit s'analyser comme une progression vers les chefsd'œuvre de la fin et donc que le coup d'essai doit être considéré comme forcément inférieur aux ouvrages qui l'ont suivi. La fin de ce texte liminaire laisse

« second ordre ». L'édition est illustrée de dessins de Le Barbier, mais certaines réimpressions proposent des gravures de Moreau le jeune.

6. CEuvres complètes de Jean Racine, avec les notes de tous les commentateurs, édition publiée par L. AiméMartin, Paris, Lefèvre, 1820, 6 vol. in- $8^{\circ}$. L'édition (qui connaîtra de très nombreuses réimpressions) est illustrée de dessins de Moreau le jeune.

7. Tous les éditeurs postérieurs en feront grand usage. Ils utiliseront aussi, sans toujours le dire, les comparaisons entre les tragédies grecques et leurs «imitations» françaises développées par le père Brumoy dans son monumental Théâtre des Grecs (1730).

8. Pierre-Joseph-François Luneau de Boisjermain (1732-1801) est surtout connu pour ses démêlés avec Diderot et les éditeurs de l'Encyclopédie. Mais il fut aussi un pédagogue novateur, dans le domaine de l'enseignement des langues, et le responsable de la première édition savante de Racine, à propos de laquelle il entra en conflit avec la corporation des libraires. 
entendre, de plus, que des «fautes de toute espèce » (p. 10) entachent la première tragédie de Racine et qu'il serait lourd et fastidieux de vouloir les signaler toutes ${ }^{9}$. Entre ces deux jugements, il n'est pour ainsi dire plus question de la pièce, mais seulement des origines de son sujet: le commentateur résume de façon assez détaillée Les Phéniciennes d'Euripide et les Sept contre Thèbes d'Eschyle, avant d'évoquer rapidement l'Antigone de Sophocle, « où il n'est question que de la sépulture de Polynice », et d'exécuter la pièce de Rotrou, " grossière esquisse du sujet de La Thébaïde» dans ses trois premiers actes, et «l'ouvrage attribué à Sénèque » (p. 9), dont la perte partielle ne paraît pas vraiment regrettable.

Le lecteur de bonne foi se laisse donc convaincre aisément que Racine a, pour ses débuts, donné une tragédie médiocre imitée d'Euripide ${ }^{10}$ et d'Eschyle : l'annotation de la pièce, qui porte surtout sur les variantes des différentes éditions et sur la critique de quelques détails d'écriture et de quelques faiblesses dramaturgiques, n'est évidemment pas apte à nuancer ce point de vue initial, que l'«Examen» placé après la tragédie, qui se présente comme écrit dans la continuité des notes, semble vouloir réitérer, non sans quelques curieuses contorsions :

Il résulte de toutes ces observations, que La Thébaïde est l'ouvrage d'un écolier, mais d'un écolier de génie, qui n'a point encore approfondi ni le théâtre ni sa langue. Quoique cette pièce ne puisse pas être comparée aux chefs-d'œuvre de Racine, elle annonçait déjà un homme bien supérieur aux contemporains de Corneille (p. 141).

Autrement dit : ce débutant de génie qu'est le jeune Racine, malgré sa méconnaissance complète des règles et de la langue du théâtre, est déjà bien meilleur que ses contemporains, dont on a soin d'excepter Corneille - que Luneau de Boisjermain, tout au long de son ouvrage, s'emploie à dédouaner des reproches que lui avait faits Voltaire dans son édition de 1764. Du reste, si La Thébaïde ne permet pas encore d'admirer «ce style enchanteur et magique qu'on admire dans les autres pièces de Racine », si elle abuse des " pointes » et du « faux bel esprit », elle est écrite « avec bien plus de naturel et de vérité » que les pièces contemporaines et comporte « des vers fort heureux, des tirades bien faites, des morceaux pleins de sentiment » (p. 144).

9. «Ce ne serait pas, certes, une petite tâche que d'entreprendre d'en faire connaître toutes les fautes », écrit Fontanier dans ses Études de la langue française sur Racine (éd. citée, t. I, p. 2), qui sont d'abord une compilation commentée des remarques de tous ses prédécesseurs. Rappelons que l'abbé d'Olivet, tout le premier, avait cru préférable de fermer «les yeux sur l'essai d'un jeune homme» (Remarques de grammaire sur Racine, Paris, Gandouin, 1738, p. 8).

10. Louis Racine disait déjà - ce qui est fort contestable - que son père avait « suivi à peu près le plan d'Euripide» (Remarques sur les tragédies de Jean Racine, dans CEuvres de Louis Racine, Paris, Le Normant, 1808, t. V, p. 292). 
On assiste perplexe à une sorte de valse-hésitation critique, qui culmine dans le jugement porté sur les caractères. Celui de Jocaste « est tendre et intéressant », mais il lui manque tout de même la sensibilité qu'on attendrait d'une mère. Celui des deux frères "paraît être le même », ce qui est un défaut, mais Racine a bien fait peut-être de peindre « avec des traits vigoureux » la haine de jumeaux qui «devaient être semblables de traits» (p. 141)... Quant à celui de Créon, il est contestable au point que le commentateur, après avoir tenté de le défendre du reproche d'incohérence en prétendant le présenter tel que Racine l'a conçu, ne changeant de langage que «suivant les intérêts des acteurs devant lesquels il parle », approuvant « la paix devant Antigone et Jocaste », prônant la guerre «devant Étéocle » mais demeurant stable dans «sa politique » et «son ambition» (p. 142), n’hésite pas à l'anéantir en présentant ce que Racine « aurait fait» s'il avait traité ce sujet dans sa maturité ! Cette proposition est d'autant plus intéressante qu'elle correspond à une procédure dramaturgique très répandue au siècle des Lumières. D’après Luneau de Boisjermain ou son collaborateur $^{11}$, Créon aurait dû être le pivot de la pièce et, au lieu de tirer les marrons du feu en " heureux ambitieux », il aurait dû manipuler les autres personnages et agir sur les événements pour s'emparer du trône :

Il aurait d'abord instruit le spectateur du dessein où il était d'armer les deux frères l'un contre l'autre : par là, le langage différent que ce prince artificieux aurait été obligé de tenir, loin d'être une contradiction dans sa conduite, n'aurait été qu'un moyen plus naturel de parvenir à ses fins. Jocaste, au lieu de soupçonner la perfidie de ce prince, se serait expliquée devant lui sur tout ce qu'elle aurait imaginé de plus propre à rétablir l'union entre ses enfants ; cette connaissance aurait fourni à Créon les moyens de traverser les démarches de cette mère infortunée [...]. Jocaste aurait toujours intéressé en faveur de sa tendresse et de l'erreur où l'aurait jetée sa confiance. [...]. Racine se serait bien gardé de défigurer le rôle de ce prince par un amour aussi ridicule que peu vraisemblable (p. 142-143).

Inutile de souligner que cette reconstruction est, en réalité, une démolition en règle du nœud développé par Racine. Et le commentateur, emporté par son élan, élargit le propos. De Créon, il passe à "l'épisode inutile de Ménécée », dont il eût fallu faire l'économie, à «l'intrigue puérile et fade d'Antigone et d'Hémon », dont il eût fallu « dégager la pièce », au caractère des jumeaux, qu'il eût fallu contraster en donnant à Polynice «un caractère doux, un courage noble », face à «l'humeur altière » et à «la confiance orgueilleuse » (p. 143) d'Étéocle.

11. Il y a de fortes chances que cette reconstruction du sujet de La Thébaïde soit due à AdrienMichel-Hyacinthe Blin de Sainmore (1733-1807), poète et auteur de la tragédie d'Orphanis (1773). 


\section{La Harpe ${ }^{12}$ (1807)}

Même s'il ne manque pas l'occasion de s'inscrire en faux contre "l'ancien commentateur» - c'est ainsi qu'il désigne Luneau de Boisjermain et ses collaborateurs -, La Harpe, d'après la " Préface du nouvel éditeur » ${ }^{13}$ placée en tête de La Thébaïde dans le tome I des CEuvres complètes, ne se montre pas moins réservé à l'encontre de la première tragédie de Racine : « Cette pièce, estime-t-il, n'annonçait que du talent pour la versification, et fort peu de talent dramatique » (p. 149). Encore émet-il de sérieuses réserves : à côté de quelques passages incontestablement réussis, "tous les défauts de goût et de style, dont Corneille n'avait pas purgé la scène » entachent une pièce où abondent "la froide recherche des idées subtiles, les locutions familières, la maladroite jactance du crime et le ton de la galanterie romanesque » (p. 150). Bref, pour lui, Racine ne s'est pas contenté d'«imiter les défaut de Corneille» (p. 151), il a surenchéri de beaucoup sur eux, parvenant même à défigurer par l'excès d'espritit le pathétique du rôle de Jocaste.

C'est cependant sur le rôle de Créon que porte l'essentiel de la critique ${ }^{15}$. Racine a donné à ce personnage «la puérile rhétorique des scélérats qui se glorifient de l'être » et cette " galanterie fade que Corneille croyait bérö̈que » ${ }^{16}$ (p. 151). La virulence indignée et sarcastique du commentaire mérite une longue citation :

Il faut avouer que jamais ce travers ne fut plus inconcevable et plus révoltant que dans le Créon de La Thébaïde, qui tout à coup au cinquième acte, après la mort de ses deux fils, après le meurtre de ses deux neveux qui se sont entretués, après la mort de Jocaste qui s'est poignardée, s'avise de proposer sa main à Antigone, la maitresse de l'un de ses fils, et l'entretient de son amour et de son mariage avec un sérieux qui fait rire. Ce n'est pas tout : après que cette Antigone s'est tuée aussi pour toute réponse, ce même Créon, qui jusque-là n'avait été qu'un politique ambitieux, qui même se piquait d'être fort peu sensible à la perte de ses fils, parce qu'il gagnait un trône; ce Créon, qui s'embarrasse peu de ce qu'exige de lui le rang de père, parce qu'il obtient celui de roi, et que la terre a moins de rois que le ciel n'a de dieux ${ }^{17}$; qui pendant quatre actes n'a pas dit un mot de ce

12. Jean-François de La Harpe (1739-1803), poète, auteur tragique, journaliste (notamment au Mercure), fut d'abord un émule de Voltaire. À partir de 1786, il professa un cours de littérature au Lycée, institution qui annonçait, à certains égards, nos modernes universités du temps libre. Emprisonné sous la Terreur après avoir été un ardent révolutionnaire, il renia ses attachements antérieurs et devint aussi acharné à décrier le parti philosophique qu'il avait été zélé à en épouser la cause. Son Lycée ou Cours de littérature ancienne et moderne, paru à partir de 1799, est l'ouvrage fondateur de l'histoire littéraire moderne.

13. Une note précise que cette préface tiendra lieu d'examen (p. 149).

14. «Son premier penchant l'entraîne vers le bel esprit », reconnait Louis Racine (Remarques sur les tragédies de Jean Racine, éd. citée, p. 300).

15. Louis Racine lui-même développe sur une page entière la « critique » de Créon (Remarques sur les tragédies de Jean Racine, éd. citée, p. 297-298).

16. Souligné dans l'édition utilisée.

17. Voir La Thébaïde, acte $\mathrm{V}$, scène 4 (Créon à son confident Attale). 
bel amour, tombe subitement dans des transports de désespoir et de rage amoureuse, encore plus plaisants que tout ce qui précède ; et pour tout dire enfin, le style est digne du fond, et le tout formerait une excellente parodie (p. 151-152).

Oui, décidément, en conclut le «nouvel éditeur », Racine, qui trois ans plus tard devait être l'auteur de « cette admirable Andromaque » (p. 152), est parti de bien bas! Et c'est essentiellement parce qu'il n'avait pas encore bien compris que certains «sujets dramatiques », notamment ceux tirés du cycle des Labdacides, ne sont pas susceptibles de faire place à une histoire d'amour ${ }^{18}$.

À partir de là, La Harpe se déchaîne, énumérant pour les récuser toutes les « conceptions dramatiques » de l'auteur des Frères ennemis : les rôles d'Antigone et d'Hémon, dont les amours sont « de la plus mortelle froideur», sont «absolument inutiles »; l'épisode de Ménécée « est aussi mal conçu que mal amené »; la tragédie est " dénuée d'action et de mouvement» (p. 154); l'absence de différenciation des caractères de Polynice et d'Étéocle est une faute. Racine, donc, n'a pas su tirer parti du modèle fourni par Euripide dans ses Phéniciennes, que le critique commente et analyse en insistant particulièrement sur l'unité d'intérêt qui fédère la pièce autour de l'entrevue entre les deux frères ennemis, sur l'«attendrissement » que le poète a su ménager et sur l'usage qu'il a fait de la suspension et des « ressources du spectacle» (p. 157): toutes ces qualités sont absentes de La Thébaïde moderne, dans laquelle du reste le jeune dramaturge ne pouvait guère s'appuyer sur ses autres prédécesseurs, Eschyle n'ayant laissé qu'un fragment d'épopée au lieu d'une tragédie, Rotrou ayant cousu « une mauvaise copie » des « deux bons originaux» (p. 157) que lui fournissaient Euripide et Sophocle et la pièce attribuée à Sénèque ne méritant même pas qu'on en parle.

On voit que la critique de La Harpe demeure en somme assez proche de celle de Luneau de Boisjermain, accablé dans la conclusion de la "Préface du nouvel éditeur » sous les sarcasmes, notamment pour avoir osé proposer un plan alternatif à celui suivi par Racine, mais imité dans son choix de ne pas multiplier les annotations sur une tragédie dont «la diction est trop vicieuse pour comporter un commentaire complet» (p. 159-160). Le lecteur ne peut pas manquer de se laisser convaincre que La Thébaïde est une mauvaise pièce, mal écrite et mal conçue, dans laquelle le dramaturge débutant n’a pas su tirer parti des ressources fournies par le seul bon modèle antique qu'il avait à sa disposition, celui d'Euripide. C'est là, du reste, la principale différence entre la lecture du « nouvel éditeur» et celle de l'« ancien commentateur », qui semblait suggérer que Racine avait puisé largement à la source grecque.

18. Dans ses Remarques sur les tragédies de son père, Louis Racine discute la question de la place de l'amour dans La Thébaïde, estimant évidemment que le poète aurait dû s'abstenir de faire intervenir ce sentiment, mais qu'il ne pouvait guère s'en dispenser, tant était grande la pression du goût du public pour la galanterie (voir les Remarques sur les tragédies de Jean Racine, éd. citée, p. 292). 
Dans le Lycée ou Cours de littérature ancienne et moderne, qui ne consacre que très peu d'espace aux Frères ennemis - La Harpe semble préférer cette désignation à celle de Thébaïde -, le jugement, quoique sévère, est peut-être un peu moins agressivement négatif ${ }^{19}$ : le critique relève que les deux frères, «à peu près également coupables, également odieux », ne peuvent pas éveiller l'intérêt du spectateur, non plus que la «douleur impuissante » de leur mère ou les « intrigues d'amour » déplacées « au milieu des horreurs de la race de Laïus » ${ }^{20}$. Il attaque aussi assez railleusement le caractère de Créon, dont l'amour pour Antigone est comparé à celui de Maxime pour Émilie dans le Cinna de Corneille, reproche à Racine l'abus des monologues sans nécessité et l'usage archaïque des stances, déplore la confusion entre « raisonnements alambiqués » et sentiments authentiques. Mais il cite avec éloge quelques vers de Polynice ${ }^{21}$, «d'une tournure ferme et d'un grand sens », et d'autres d'Étéocle 22 , «d'un style tragique» : à ses yeux, du moins à ce moment-là, « ce coup d'essai n'est pas sans beautés » ${ }^{23}$.

\section{Geoffroy $^{24}$ (1808)}

Ce n'est pas un simple « examen » que Geoffroy, au tome I de son édition des Euvres de Racine avec un commentaire, consacre, sous le titre de « Jugement sur La Thébaïde et sur les auteurs qui ont traité le même sujet », à la première tragédie de Racine. Sur plus de cinquante pages, il déploie une vaste érudition et analyse, avec force citations, l'essentiel des pièces anciennes et modernes qui ont traité le sujet des Frères ennemis, jusqu'à l'Étéocle de Legouvé (1799). On se limitera à souligner ce qui est comparable aux propos de Luneau de Boisjermain et de La Harpe dont on a fait mention jusqu'ici, qui est rassemblé dans le préambule de ce «Jugement » dont la longueur est assez comparable aux examens des autres critiques.

D’emblée, Geoffroy déclare que le jeune Racine, à l'époque de ses débuts, n'était pas capable de «combiner un plan, créer des situations et maîtriser son

19. Peut-être parce que le critique réutilise prioritairement le matériau de son Éloge de Racine (1772), rédigé pour un concours de l'Académie de Marseille. La section consacrée à Racine dans le Lycée s'ouvre d'ailleurs sur une longue citation - identifiée comme telle - de cet Éloge, reproduit (sans ses notes) dans l'édition des Euvres complètes, en 1807, en guise d'introduction générale.

20. Lycée ou Cours de littérature ancienne et moderne (publication originale à partir de 1799), Paris, Déterville, 1818, t. V, p. 302.

21. Tirés de l'acte II, scène 3 (Polynice à Jocaste) : «C'est un tyran qu'on aime, / Qui par cent lâchetés tâche à sa maintenir...»

22. Tirés de l'acte IV, scène 1 (Étéocle à Créon) : «Je ne sais si mon cœur s'apaisera jamais... »

23. Lycée..., éd. citée, p. 304.

24. Julien-Louis Geoffroy (1743-1814), d'abord Jésuite, fut professeur au collège de Navarre et au collège des Quatre-Nations. Collaborateur de l'Année littéraire après la disparition de Fréron (1776), il eut une activité journalistique intense et variée, notamment au service du parti royaliste. Sous le Consulat, il rejoignit le Journal des débats (futur Journal de l'Empire), y donnant notamment une passionnante série de feuilletons dramatiques. 
sujet» (p. 127) et que, ayant choisi d'imiter ses prédécesseurs, il n'a pas su fondre ses emprunts dans sa pièce et n'a presque rien «ajouté de son propre fonds ». Il souligne que le dramaturge débutant, s’il a suivi parfois Euripide et Sénèque, s'est surtout attaché à calquer Rotrou, pour la conduite de sa tragédie, et Corneille, pour le style, gâché par l'abus des «déclamations » et des « raisonnements subtils », mais parfois traversé d'éclairs annonciateurs de l'«heureux talent pour la versification» et de l'«éloquence naturelle » dont témoigneront les productions de sa maturité créatrice :

Malgré les incorrections, les négligences et les fautes de toute espèce échappées à l'inexpérience, à la précipitation, cette pièce, comparée à celles qui paraissaient en ce temps-là, pouvait passer pour bien écrite : on y voit que le poète tend au naturel, à l'élégance, à la politesse ; qu'il commence à perfectionner la langue poétique (p. 127).

Bref, le critique estime que comparée aux productions contemporaines de Quinault et des deux Corneille La Thébaïde, sans être à la hauteur des chefsd'œuvre qui suivront, n'en est pas moins «l'ouvrage d'un grand poète ».

Ses défauts majeurs ne sont pas à chercher dans les détails du style, mais dans la mauvaise conception d'ensemble et l'écriture dramatique mal maîtrisée : l'intérêt, reposant sur deux frères qui ont « la même couleur » et sur une Jocaste «monotone », ne saurait se soutenir; le dialogue, malgré des stichomythies parfois bien conduites - que Geoffroy rapproche de la manière de Sénèque -, est plutôt fait de « longues conversations » que d'échanges « serrés et brillants ». Surtout, Racine a vraiment mal conçu le caractère de Créon :

Le rôle de Créon est froid et dégoûtant; ses amours sont insupportables, et ses folies à la fin de la pièce auraient suffi pour la faire tomber, si elles n'avaient été sous la sauvegarde particulière de la galanterie et du mauvais goût qui dominaient alors (p. 128).

Il y a donc peu à sauver dans le coup d'essai du jeune Racine ${ }^{25}$, même si sa tragédie n’est pas aussi mauvaise que le prétendent les commentateurs précédents et si l'évolution du goût et des mœurs peut lui servir d'excuse : la rivalité des jumeaux ennemis, qui était «tragique pour les Grecs », est désormais « odieuse pour des Français », qui se sauraient accepter de voir s'entredéchirer « deux bêtes farouches toujours prêtes à se dévorer». Si Racine est coupable, en somme, c'est de ne s'être pas avisé que le sujet qu'il a retenu, faisait basculer la tragédie de la terreur (acceptable) dans l'horreur (intolérable).

La suite des développements de Geoffroy n'intéresse d'abord pas directement notre sujet : il donne des « extraits » - au sens de l'époque : des analyses

25. «Quelques tirades d'Étéocle et de Polynice, certains morceaux de Jocaste, et surtout la scène de l'entrevue des deux frères au quatrième acte $[.$.$] ; enfin le récit du combat des deux$ frères, voilà ce qui est digne de la jeunesse de Racine... » (p. 128). 
accompagnées d'amples citations, traduites au besoin par ses soins d'Euripide, de Sénèque et de La Thébaïde de Stace. Mais il en vient à examiner scrupuleusement l'Antigone de Rotrou ${ }^{26}$, dont « les trois premiers actes sont une imitation des Phéniciennes d'Euripide, et encore plus de La Thébaïde de Sénèque » et les derniers « une traduction de l'Antigone de Sophocle » (p. 176), pour constater la dette de Racine envers son prédécesseur :

C'est Rotrou qui a fourni à Racine le caractère de Créon, les amours d'Hémon et d'Antigone, la férocité de Polynice, la supposition que Polynice est haï des Thébains, les stances d'Antigone et presque tout ce qu'il y a de vicieux dans sa Thébaïde (p. 176-177).

C'est Rotrou encore qui a poussé Racine à se tourner vers Sénèque plutôt que vers Euripide... Autre excuse, si l'on veut, pour les fautes commises : le choix d'un mauvais guide!

Tout ce commentaire, qui se termine par un survol du Polynice d'Alfieri que Geoffroy juge avec une relative indulgence - et de l'Étéocle de Legouvé auquel il reproche sa froideur et la faiblesse de son style -, aboutit à ce jugement péremptoire :

Ainsi, en exceptant Euripide, qu'un auteur si jeune ne pouvait encore égaler, Racine est celui qui a fait sur ce sujet, essentiellement mauvais pour nous, la tragédie la moins mauvaise (p. 185).

Le lecteur de bonne fois, quant à lui, est bien obligé de comprendre que, malgré cette hiérarchisation favorable au jeune dramaturge français, Racine débutant n'a pas su se tourner décidément vers les véritables sources antiques de la tragédie, ce qui est tout à fait conforme à la thèse majeure du commentaire de Geoffroy, qui perçoit l'évolution racinienne comme une progression vers une compréhension de plus en plus profonde de la source grecque qui finit par le conduire, au moment d'Iphigénie et de Phèdre, à devenir le digne rival d'Euripide.

\section{$\operatorname{Aignan}^{27}$ (1824)}

Au tome I des Euvres complètes de J. Racine publiées par Aignan, La Thébaïde est suivie, selon une procédure inaugurée dans l'édition de peu antérieure due à

26. On remarquera que Geoffroy se refuse à considérer l'Antigone de Garnier, « qui écrivait au sein de la barbarie» (p. 176). On signalera aussi que la grande édition des CEuvres de Rotrou donnée chez Desoer par Viollet-Le-Duc n'est parue qu'en 1820.

27. Étienne Aignan (1773-1824) a été haut-fonctionnaire sous l'Empire. Son œuvre dramatique, écrite à partir de 1793, est relativement abondante, mais il est surtout connu pour ses ouvrages juridiques et politiques - publiés sous la Restauration, époque où il déploya une grande activité de journaliste dans le camp libéral - et pour sa traduction en vers de l'Iliade, dans laquelle il témoigne d'une grande érudition. 
Aimé-Martin ${ }^{28}$, par une section développée (pas loin de trente pages de typographie serrée) consacrée à la "Traduction des passages d'Euripide, de Sénèque et de Stace imités par Racine ». Ce n'est qu'après qu'est placée une brève "Étude ", complaisamment rédigée, qui passe d'abord en revue les principaux prédécesseurs de Racine: le critique, qui semble préférer la "sévère production » d'Eschyle à la tragédie écrasée "sous les accessoires» (p. 284) d'Euripide, cite abondamment l'Antigone de Rotrou, qui lui sert notamment à polémiquer - nous sommes au début des batailles romantiques - contre «les pièces étrangères dont les importations sont si multipliées » et lui parait avoir souvent « égalé Corneille».

Son jugement sur La Thébaïde elle-même est sensiblement moins sévère que celui de ses prédécesseurs : la fable en est conduite «avec une habileté remarquable ${ }^{29}$; certaines péripéties (la trêve de la fin du premier acte, la « rupture, imitée de l'Iliade » de la fin du deuxième) sont « excellentes »; « la belle entrevue des deux frères » et «la catastrophe dont elle est la suite nécessaire » méritent "les éloges généralement donnés au plan de ce premier essai d'un grand poète » (p. 285). Aignan défend même les caractères de la pièce : si Créon apparaît " comme bassement odieux », si Jocaste et Antigone n'emportent pas absolument l'adhésion, c'est seulement parce que le poète débutant n'est pas parvenu à leur donner «le coloris » adéquat. Même les jumeaux lui semblent être «ce qu'ils doivent être, c'est-à-dire atroces, effrénés, tels que deux frères qui finiront pas s'égorger l'un l'autre » (p. 288). Il n'approuve donc pas ceux qui ont proposé de contraster les caractères respectifs d'Étéocle et de Polynice. Pas non plus la transformation de Créon en un manipulateur machiavélique.

Le lecteur est donc invité à considérer la première tragédie de Racine comme un premier chef-d'œuvre, ce qu'aucun des commentateurs antérieurs n'osait vraiment proposer : aux côtés des Sept contre Thèbes d'Eschyle et du début de l'Antigone de Rotrou, elle lui semble constituer la meilleure version disponible de ce sanglant épisode de l'histoire des Labdacides.

$* * *$

On le voit: malgré ce dernier jugement plus favorable que ceux qui l'ont précédé, les premiers éditeurs savants des tragédies de Racine portent sur $L a$

28. Voir ci-dessus note 6. Louis Aimé-Martin (1782-1847) fut professeur à l'École polytechnique sous l'Empire, puis conservateur de la Bibliothèque Sainte-Geneviève sous la Restauration. Auteur de quelques essais dramatiques et des Lettres à Sophie sur la physique, la chimie et l'bistoire naturelle (1811), ouvrage de vulgarisation mondaine dans lequel le texte est entremêlé de morceaux en vers, il se fit l'éditeur de nombreux classiques français à partir des années 1820, mais aussi de Bernardin de Saint-Pierre, dont il avait épousé la veuve.

29. C'est ce que disait déjà Louis Racine : «L'économie de la tragédie que j’examine annonce un poète qui entend bien à conduire un sujet » (Remarques sur les tragédies de Jean Racine, éd. citée, p. 299). 
Thébaïde, en des termes plus ou moins nuancés, un regard relativement sévère. Quelques beaux morceaux n'y rachètent pas une intrigue imparfaitement constituée, des caractères mal conçus et un style entaché de baroquismes. Mais ce n'est peut-être pas là l'essentiel : à travers leurs commentaires - et l'étude précise des annotations qui se stratifient presque à l'infini au fil des éditions le confirmerait probablement -, c'est un ensemble de questions qui sont posées, dont l'utilité même pour le lecteur d'aujourd'hui semble difficilement contestable. La principale, à nos yeux, est celle de l'imitation, qui recouvre en partie celle de l'esthétique de la pièce : quel modèle Racine a-t-il choisi de suivre? Euripide? Sénèque? Rotrou ? Corneille? Sa tragédie est-elle un premier pas résolu sur les chemins de la Grèce ou bien demeure-t-elle fortement tributaire de la tradition baroque?

Les éditeurs savants, entre 1760 et 1825, nous semblent avoir eu l'intuition de la pertinence de ce questionnement : si Luneau de Boisjermain et même La Harpe optent - fût-ce pour en souligner l'échec - pour la première réponse, Geoffroy (que ses positions esthétiques conduisent à la considérer comme à l'origine du demi-échec de la pièce) et Aignan (qui lui fait jouer, notamment, un rôle stratégique face à la fascination éprouvée par le romantisme naissant vis-àvis de l'étranger) ont visiblement pressenti l'intérêt de la seconde ${ }^{30}$.

30. On aurait bien tort de négliger les ouvrages déjà anciens de Roy Clement Knight (Racine et la Grèce, Paris, Boivin, 1950) et de Philip Butler (Classicisme et baroque dans l'auvre de Racine, Paris, Nizet, 1959). 